\title{
Discussing Recent Research on Sensory Communication in Marketing: An Abstract
}

\author{
Klaus-Peter Wiedmann, Janina Haase, and Jannick Bettels
}

\begin{abstract}
The ever-growing number of products with similar qualities in combination with decreasing impacts of conventional marketing techniques has led to increasing difficulties for marketing practitioners to appeal to the consumer effectively. As a consequence, sensory marketing has recently gained growing popularity with respect to the ongoing search for new ways to differentiate products and brands from competitors. Several research results have already provided evidence for the great potential of sensory communication in marketing by investigating the effects of for instance the salience of touch, store scents, and background music on consumer behavior. However, there are still numerous unexplored topics in this broad research field. Therefore, we contribute to this promising stream of research by discussing our results of three recent studies investigating several new aspects of sensory communication in marketing.
\end{abstract}

References Available Upon Request

K.-P. Wiedmann $(\bowtie) \cdot$ J. Haase $\cdot$ J. Bettels

Leibniz University of Hannover, Hannover, Germany

e-mail: wiedmann@m2.uni-hannover.de; haase@m2.uni-hannover.de;

bettels@m2.uni-hannover.de 